\title{
Special Issue on Wearable Computing and Machine Learning for Applications in Sports, Health, and Medical Engineering
}

\author{
Sunghoon I. Lee ${ }^{1, *}$ and Bjoern M. Eskofier ${ }^{2, *}$ (1) \\ 1 College of Information and Computer Sciences, University of Massachusetts Amherst, \\ Amherst, MA 01002, USA \\ 2 Department of Computer Science, Friedrich-Alexander University Erlangen-Nuernberg, \\ 91054 Erlangen, Germany \\ * Correspondence: silee@cs.umass.edu (S.I.L.); bjoern.eskofier@fau.de (B.M.E.)
}

Received: 22 January 2018; Accepted: 23 January 2018; Published: 25 January 2018

\section{Introduction}

Recent advancement in digital technologies is driving a remarkable transformation in sports, health, and medical engineering, aiming to achieve the accurate quantification of performance, well-being, and disease condition, and the optimization of sports, clinical, and therapeutic training and treatment programs. Traditionally, understanding and monitoring of functional performance and capacity has been performed in gait laboratories based on optoelectronic motion capture systems. However, gait laboratories in practical settings are often not readily available because the systems are costly and require trained experts to operate. Most importantly, when assessments are restricted to laboratory settings, they provide a narrow snapshot of function and do not capture functionality in natural free-living settings, thus representing a severely under-sampled view of an individual's condition. The use of mobile and wearable technologies has been explored in many sports, health, and medical research studies examining individuals in "in-the-wild" settings. Among the most important drivers of this transformation are (1) wearable sensors and (2) signal processing and machine learning algorithms. Wearable sensors are capable of collecting physical and/or physiological data continuously and seamlessly outside of laboratory settings. Signal processing and machine learning algorithms allow data-driven approaches for analyzing considerable amounts of multidimensional sensory data and for extracting important information relevant to the mentioned application areas (e.g., validating the efficacy of sports training, health benefits, and chronic disease progression). These technologies together would support how sports and clinical professionals understand and interpret individuals' performance more objectively, and enable proactive, evidence-based, and personalized management systems.

\section{Wearable Computing and Machine Learning for Applications in Sports, Health, and Medical Engineering}

This special issue was introduced to collect the latest research on wearable computing and machine learning for applications in sports, health, and medical engineering. A total of 14 papers were submitted to this special issue and six of them were published ( $43 \%$ acceptance rate). All papers have gone through a rigorous peer-review procedure based on the editorial policy of Applied Sciences. The special issue spanned a wide range of topics relevant to wearable computing, including one review article and five research articles.

The review paper, authored by B. M. Eskofier et al., presented an overview of the use of smart shoes in Internet of Things (IoT) environments [1]. This paper focused on providing a comprehensive review on gait and mobility assessment-which are of the utmost importance as they define quality 
of life in healthy living and chronic diseases-using a different type of smart shoes. This special issue also included two research papers related to gait and locomotion monitoring based on wearable sensors. The first paper, 'Which Method Detects Foot Strike in Rearfoot and Forefoot Runners Accurately when Using an Inertial Measurement Unit?' authored by C. Mitschke, T. Heß, and T. L. Milani, investigated different algorithms to detect foot strike (i.e., the moment when the foot first touches the ground) using six-axis inertial units [2]. Specifically, authors evaluated the efficacy of the considered algorithms in different practical experiment setups where footwear hardness and locomotion patterns were varied. The second paper, 'A Single Gyroscope Can Be Used to Accurately Determine Peak Eversion Velocity during Locomotion at Different Speeds and in Various Shoes' authored by C. Mitschke, M. Öhmichen, and T. L. Milani, introduced a method to measure the frontal plane peak angular velocity during locomotion using a single gyroscope [3].

This special issue published three articles that introduced cutting-edge wearable technologies related to underwater electrocardiogram (ECG) monitoring, hip fracture prevention, and communication for deaf-blind users. The first paper, 'Wearable Current-Based electrocardiogram (ECG) Monitoring System with Non-Insulated Electrodes for Underwater Application' authored by S. Gradl et al., introduced a novel, wearable, current-based ECG device that allows underwater signal acquisition without having to use insulated electrodes [4]. The second paper, 'A Biomechanical Study for Developing Wearable-Sensor System to Prevent Hip Fractures among Seniors' authored by G. Shan, X. Zhang, M. Meng and B. Wilde, introduced a wearable system that can prevent hip fracture by triggering a mini-airbag prior to fall events [5]. Lastly, 'Sensing Performance of a Vibrotactile Glove for Deaf-Blind People' authored by A. Carrera et al., introduced a vibrotactile glove that can assess the viability of communication between a deaf-blind user and his/her interlocutor [6].

In summary, this special issue introduced a variety of novel wearable sensors for applications in sports, health, and medical engineering. Specifically, significant progress was being made to push the applications in gait, mobility, and fall prevention, which are important topics of study in geriatrics. We hope that the studies presented in this special issue will guide future efforts in the field of wearable computing toward the development of high-quality systems to further expand their applications in various practices.

Acknowledgments: This issue would not be possible without the contributions of authors, reviewers, and editorial team of Applied Sciences. We would like to congratulate and thank all authors who dedicated their efforts to introduce cutting-edge technologies. We also would like to thank all reviewers who provided valuable comments, feedback, and suggestions to the published papers. Finally, we are grateful to the support provided by the editorial team of Applied Sciences with special thanks to Felicia Zhang, Assistant Managing Editor.

Conflicts of Interest: The authors declare no conflict of interest.

\section{References}

1. Eskofier, B.M.; Lee, S.I.; Baron, M.; Simon, A.; Martindale, C.F.; Gaßner, H.; Klucken, J. An Overview of Smart Shoes in the Internet of Health Things: Gait and Mobility Assessment in Health Promotion and Disease Monitoring. Appl. Sci. 2017, 7, 986, doi:10.3390/app7100986.

2. Mitschke, C.; Heß, T.; Milani, T.L. Which Method Detects Foot Strike in Rearfoot and Forefoot Runners Accurately when Using an Inertial Measurement Unit? Appl. Sci. 2017, 7, 959, doi:10.3390/app7090959.

3. Mitschke, C.; Öhmichen, M.; Milani, T.L. A Single Gyroscope Can Be Used to Accurately Determine Peak Eversion Velocity during Locomotion at Different Speeds and in Various Shoes. Appl. Sci. 2017, 7, 659, doi:10.3390/app7070659.

4. Gradl, S.; Cibis, T.; Lauber, J.; Richer, R.; Rybalko, R.; Pfeiffer, N.; Leutheuser, H.; Wirth, M.; von Tscharner, V.; Eskofier, B.M. Wearable Current-Based ECG Monitoring System with Non-Insulated Electrodes for Underwater Application. Appl. Sci. 2017, 7, 1277, doi:10.3390/app7121277. 
5. Shan, G.; Zhang, X.; Meng, M.; Wilde, B. A Biomechanical Study for Developing Wearable-Sensor System to Prevent Hip Fractures among Seniors. Appl. Sci. 2017, 7, 771, doi:10.3390/app7080771.

6. Carrera, A.; Alonso, A.; de la Rosa, R.; Abril, E.J. Sensing Performance of a Vibrotactile Glove for Deaf-Blind People. Appl. Sci. 2017, 7, 317, doi:10.3390/app7040317.

(C) 2018 by the authors. Licensee MDPI, Basel, Switzerland. This article is an open access article distributed under the terms and conditions of the Creative Commons Attribution (CC BY) license (http:/ / creativecommons.org/licenses/by/4.0/). 